\title{
Microvascular Decompression of the Optic Nerve
}

\author{
Jack Andrew S ${ }^{1}$, Mc Dougall Cameron ${ }^{1 *}, 0$ Kelly Cian J ${ }^{1}$, Edwards Marianne C ${ }^{2}$, Chow, MichaelM ${ }^{1}$ \\ ${ }^{1}$ Division of Neurosurgery, Department of Surgery, 2D1 WMC, University of Alberta \\ Edmonton, Alberta, Canada \\ ${ }^{2}$ Department of Ophthalmology, University of Alberta, Edmonton, Alberta, Canada
}

Received: June 15, 2016; Accepted: July 21, 2016; Published: September 4, 2016

*Corresponding author: Cameron M McDougall, Department of Neurosurgery: University of Texas Southwestern 2728 McKinnon Street Suite 1201, Dallas, Texas, 75201, Tel no: 916-350-0635; Email: Cameron.mcdougall@gmail.com

\begin{abstract}
Symptomatic compression of the optic apparatus by a tortuous internal carotid artery (ICA) is rare. We report a patient with visual decline due to optic nerve compression by the ICA. The patient presented with a visual field deficit and standard work-up revealed no discernible cause. Magnetic resonance imaging (MRI) demonstrated distortion of the optic nerve by the ICA. The optic nerve was decompressed with resultant clinical improvement. Optic nerve compression by the ICA may be considered a diagnosis of exclusion. Here, microvascular decompression led to improvement in visual function.
\end{abstract}

Key Word: Internal carotid artery; Dolichoectasia; Microvascular decompression; Optic neuropathy

\section{Introduction}

Optic neuropathy resulting from cerebrovascular compression is rare. When seen, it is often in the context of carotid dolicoectasia. Cranial neuropathy associated with arterial pulsation is well described in Trigeminal neuralgia, hemi-facial spasm, Glossopharyngeal neuralgia and various other neuropathies [9]. Here, we describe a case of carotid artery dolichoectasia leading to a progressive visual field deficit in which work-up for other etiologies was negative.

\section{Materials and Methods}

A 36-year-old female presented with acute painless, right monocular visual field loss. She had a 12 pack-year smoking history, but was otherwise healthy at the time of presentation.

Examination revealed a dense right monocular nasal visual field deficit confirmed by Humphrey Matrix Frequency Doubling Technology (FDT) 30-2 visual field testing (figure 1a). Laboratory investigations and neuro-ophthalmological work-up revealed no definitive explanation for the patient's symptoms and included a comprehensive stroke work-up. MRI, magnetic resonance angiography, and catheter angiography revealed a tortuous, supraclinoid segment of the ICA mildly compressing and displacing the right optic nerve (figure 2).

Because of the acuity of the presenting symptoms an ischemic insult was felt to be the most likely cause of the visual field loss. The patient was managed medically with continued neuroophthalmological follow-up arranged. Her visual field deficit progressively worsened over the following 3 months which was objectively confirmed with repeat visual testing (figure $1 \mathrm{~b}$ ).

A right pterional craniotomy was performed with adequate exposure of the ICA under the operating microscope. The site of compression was the ophthalmic carotid segment where unusually dense arachnoidal adhesions were found tethering the two together (figure 3a). The adhesions were divided, and two pieces of Teflon felt were placed between the artery and nerve (figure $3 b$ ).

\section{Results}

The patient had an uncomplicated post-operative course and was discharged home four days after the operation. Notably the patient reported subjective improvement in her peripheral vision almost immediately. She continued to note this improvement at

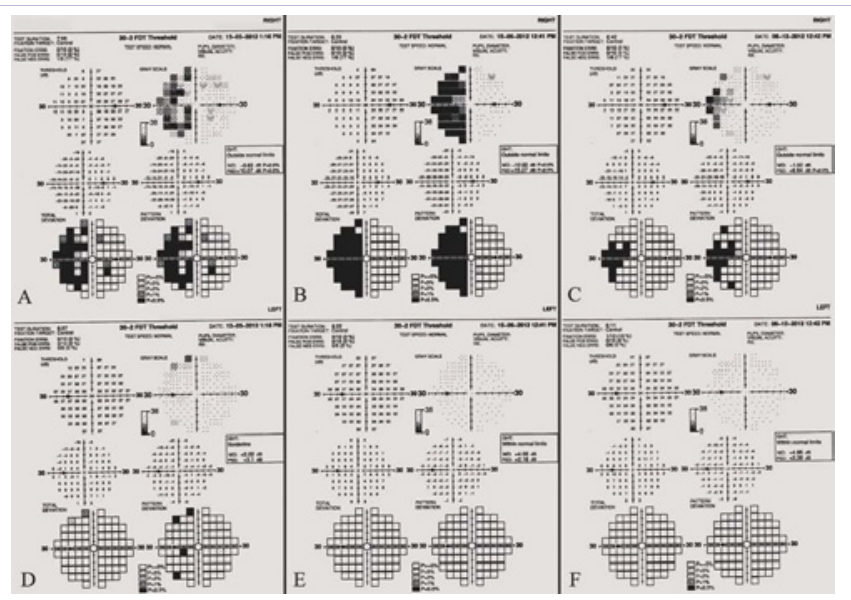

Figure 1: Matrix FDT 30-2 visual field testing results. Image (A): a dense right nasal monocular visual field deficit seen pre-operatively. Image (B): visual field testing after clinical follow-up demonstrating progression of the visual deficit. Image (C): improvement of the visual field deficit post-operatively. Images D-F demonstrates left eye visual field results from each respective examination. 
her routine 6-week follow-up, and upon repeat visual field testing was found to have objectively improved (figure 1c).

\section{Discussion}

Vascular ectasia has been found to be the cause of different visual field deficits due to compression of the optic nerve, chiasm and tract $[3,5,7]$. The visual loss is often gradual but may be precipitous and fundoscopy may be normal or reveal optic disc edema, cupping, or atrophy $[3,15]$. As a result, its diagnosis can be challenging. Patients are often followed long-term with deficits attributed to other causes such as glaucoma [13]. Analysis of patients with normotensive glaucoma, a condition characterized by visual field loss despite normal intraocular pressure, has revealed that some cases may actually be related to carotid ectasia and pulsatile demyelination of the optic apparatus [10]. Visual improvement following microvascular decompression has been shown by some $[3,7,14,15]$. This case highlights an atypical presentation of optic neuropathy due to carotid artery pulsations in a young female with resultant visual field improvement following microvascular decompression.

In contrast to most reports, the patient's pattern of vision loss was much more acute, she was substantially younger than patients in the previous reports whom also typically have numerous cardiovascular risk factors. Why a chronic process would manifest with acute symptoms is unknown, but could relate

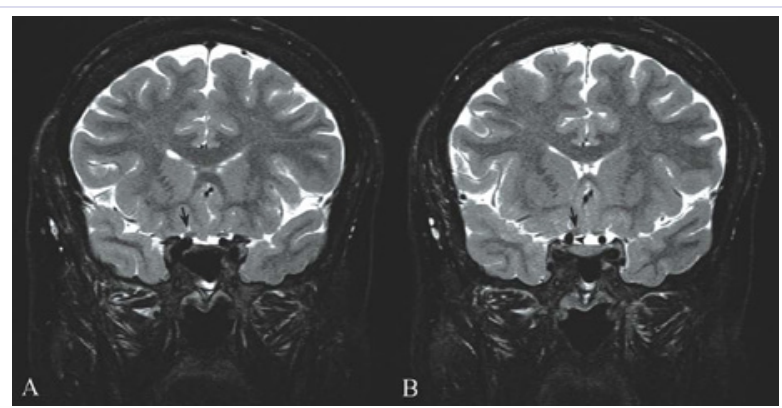

Figure 2: Coronal T2 weighted MRI depicting the right supraclinoid ICA contacting and mildly distorting the right optic nerve. Image $(A)$ is slightly more anterior than image (B) with an arrow depicting the optic nerve distortion and arrowhead showing the supraclinoid ICA

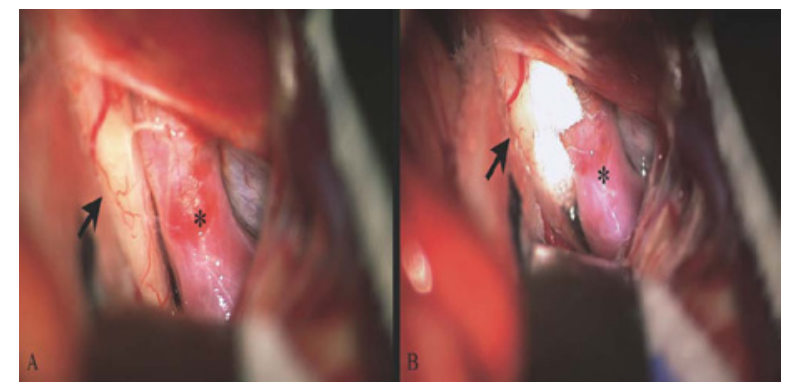

Figure 3: Operative photographs of a pterional craniotomy for microvascular decompression of the right optic nerve. Image (A): the right supraclinoidal ICA contacting and minimally distorting the adjacent optic nerve (arrow displaying the optic nerve and '*' depicting the ICA). Image (B): the optic nerve and carotid artery with felt placed between to patient factors such as initial compensation for a progressive deficit. Alternatively, the disease process itself may also explain the abrupt change with venous insufficiency in the vasa nervosum leading to micro-infarction superseded on a background of chronic demyelination. In addition, her visual deficit was nasal as opposed to the temporal deficits seen in other reports $[3,6$, $8,15]$. A nasal deficit makes more anatomical sense given the lateral location of the ICA with respect to the Optic nerve, causing preferential damage to the temporal fibers (thus affecting the nasal field). Clinical correlation such as this is important given that the imaging investigations and intra-operative findings were not completely conclusive for arterial compression and pulsatile injury of the optic nerve.

Indeed, the idea of arterial pulsations causing optic neuropathy has been considered in the past [14]. It is based on the theory of arterial compression leading to a demyelinating lesion. This allows for the ephaptic neuronal transmission seen in trigeminal neuralgia $[11,12]$. It may be that in the case of the optic nerve, demyelination leads to a visual deficit (as in optic neuritis secondary to multiple sclerosis) and that physical dampening of the arterial pulsations with felt and not necessarily "decompression" interrupts the pathophysiological mechanism underlying the visual loss. This is also similar to posterior communicating artery aneurysmal compression of the third nerve. Endovascular treatment of the aneurysm with coil occlusion does not change the mass effect but is thought to alleviate damage caused by arterial pulsations leading to a high rate of neurological recovery $[1,2,4]$.

In this case there were unusually thick arachnoid bands tethering the optic nerve to a tortuous ICA but what prevents this incredibly rare pathology from occurring more frequently remains unknown. It cannot be over emphasized that visual field loss attributed to cerebrovascular compression is a diagnosis that must be made both cautiously, and as a collaborative effort between neurosurgery, neuro-ophthalmology, and neuroradiology. Imaging may not be helpful and other more common causes of optic neuropathy such as normal pressure glaucoma, vascular optic neuropathy, or optic neuritis must be considered first.

\section{Conclusion}

This case describes the rare occurrence of visual field loss from optic nerve injury by the carotid artery. The patient experienced objective improvement following microvascular decompression. This is a diagnosis of exclusion and should only be made from a multi-disciplinary approach after ruling out other more common pathologies. Although rare and difficult to diagnose it should be kept in the differential diagnosis because selected treatment may prevent or even reverse vision loss.

All authors certify that they have NO affiliations with or involvement in any organization or entity with any financial interest (such as honoraria; educational grants; participation in speakers' bureaus; membership, employment, consultancies, stock ownership, or other equity interest; and expert testimony or patent-licensing arrangements), or non-financial interest (such 
as personal or professional relationships, affiliations, knowledge or beliefs) in the subject matter or materials discussed in this manuscript.

The patient in this case has consented to the submission of the case report for submission to the journal.

\section{References}

1. Chalouhi N TT, Jabbour P, Dumont AS, Gonzalez, Starke RM, Gordon D, et al. Endovascular treatment of posterior communicating artery aneurysms with oculomotor nerve palsy: clinical outcomes and predictors of nerve revovery. AJNR Am J Neuroradiol. 2013;34(4):828832. doi: 10.3174/ajnr.A3294.

2. Chen PR, A-HS, Albuquerque FC, McDougall CG, Zabramski JM, Spetzler RF. Outcome of oculomotor nerve palsy from posterior communicating artery aneurysms: comparison of clipping and coiling. Neurosurgery. 2006;58(6):1040-1046.

3. Colapinto EV, Cabeen MA, Johnson LN. Optic nerve compression by a dolichoectatic internal carotid artery: case report. Neurosurgery. 1996;39(3):604-606

4. FC A. Coiling versus clipping for posterior communicating artery aneurysms associated with occulomotor nerve palsy: only time will tell. World Neurosurg. 2010;74(2-3):250-251.

5. Guirgis MF, Lam BL, Falcone SF. Optic tract compression from dolichoectatic basilar artery. Am. J. Ophthalmol. 2001;132(2):283286.

6. Matsuo K, Kobayashi S, Sugita K. Bitemporal hemianopsia associated with sclerosis of the intracranial internal carotid arteries. Case report. J. Neurosurg. 1980;53(4):566-569.
7. McLaughlin N, Bojanowski MW. Microvascular decompression of the optic chiasm. Case report. J Neurosurg. 2011;114(3):857-860. doi: 10.3171/2009.9.JNS081658.

8. Mitts MG, McQueen JD. Visual loss associated with fusiform enlargement of the intracranial portion of the internal carotid artery. J Neurosurg. 1965;23(1):33-37.

9. Moeller AR, MM. Microvascular decompression operations. Prog Brian Res. 2007;166:397-400.

10. Ogata N, Imaizumi M, Kurokawa H, Arichi M, Matsumura M. Optic nerve compression by normal carotid artery in patients with normal tension glaucoma. Br. J Ophthalmol. 2005;89(2):174-179.

11. Jannetta PJ. Arterial compression of the trigeminal nerve at the pons in patients with trigeminal neuralgia. J Neurosurg. 1967;26(1):159162.

12. Sade B LJ. Microvascular decompression for trigeminal neuralgia. Neurosurg Clin N Am. 2014;25:743-749.

13. Colapinto EV, Cabeen MA, Johnson LN. Optic Nerve Compression by a Dolichoectatic Internal Carotid Artery: Case Report (Comments). Neurosurgery. 1996;39(3):604-606.

14. Strom RG, Fouladvand M, Pramanik BK, Doyle WK, Huang PP. Progressive optic neuropathy caused by contact with the carotid artery: improvement after microvascular decompression. Clin Neurology Neurosurg. 2012;114(6):812-815.

15. Unsold R. The clinical signs and symptoms of optic nerve compression and clinical disease entities masking compressive lesions. SpringerVerlag Berlin. 1989;15-34. 